\title{
Structural and electronic properties of chemisorbed oxygen on $\operatorname{Rh}(111)$
}

\author{
M. V. Ganduglia-Pirovano and M. Scheffler \\ Fritz-Haber-Institut der Max-Planck-Gesellschaft, Faradayweg 4-6, D-14195 Berlin-Dahlem, Germany
}

(Received 13 January 1999)

\begin{abstract}
Using density-functional theory within the generalized gradient approximation, we investigate the adsorption of oxygen on $\mathrm{Rh}(111)$ as a function of coverage $\Theta$. At the coverages of 0.25 monolayer (ML) and $0.5 \mathrm{ML}$ oxygen (occupying fcc-hollow sites), the calculated atomic geometries (interlayer spacings, bond lengths, and lateral displacements) are in excellent agreement with those of previous low-energy electron-diffraction intensity analyses. We find a strong coverage dependence of the oxygen-induced buckling relaxations and a substantial overall expansion of the first interlayer spacing, reflecting a weakening of metal-metal bonds between the two outer substrate layers. The work functions of the relaxed structures are presented, and the stability of the adlayers is analyzed. We also predict that the existence of a dense $O$ overlayer $(\Theta=1 \mathrm{ML})$ with a $(1 \times 1)$ periodicity is possible, because oxygen will be incorporated as a subsurface adsorbate only when the $(1 \times 1)$ adlayer is (nearly) completed. [S0163-1829(99)10219-4]
\end{abstract}

\section{INTRODUCTION}

Understanding the nature of the interaction between oxygen and metal surfaces is crucial to elucidating the role of oxygen in a number of important technological processes such as oxidation, corrosion, and heterogeneous catalysis. The structures that are formed on transition-metal surfaces when they are exposed to oxygen vary from adlayers to oxygen penetration in the subsurface region to the formation of surface oxides, depending markedly on the partial pressure and temperature and on the orientation of the surface of the particular metal. In this paper we present results for $\mathrm{Rh}(111)$ and compare them to some published work on $\mathrm{Ru}(0001)$, the left neighbor of $\mathrm{Rh}$ in the periodic table. On $\mathrm{Rh}(111)$ and $\mathrm{Ru}(0001)$ at room temperature and low pressures, dissociative chemisorption occurs and ordered structures form. The most densely packed surfaces of rhodium and ruthenium are fairly stable against reconstruction; however, relaxations of the substrate atoms induced by oxygen adsorption can be significant. ${ }^{1,2}$ At higher coverages and temperatures, oxygen may penetrate below the surface, and/or an oxide structure might form. Both the presence of subsurface oxygen and of a surface oxide significantly alter the physical and chemical properties of metal surfaces. ${ }^{3-9}$ Recently, the oxygen adsorption on $\mathrm{Ru}(0001)$ has been investigated by density-functional theory (DFT) calculations and the stability of a highcoverage $(1 \times 1)$ structure has been predicted. ${ }^{10}$ Subsequently, the existence of this dense phase has been confirmed experimentally. ${ }^{11}$ The DFT calculations also concluded that oxygen can be built in as a subsurface adsorbate, but this would happen only after the $(1 \times 1)$ adlayer is completed. In this high-coverage adsorbate phase $(\Theta \geqslant 1)$ the $\mathrm{O}-\mathrm{Ru}$ bond strength was found to be particularly weak. It has been argued that this small oxygen binding energy is likely to enhance the rate of oxidation reaction on $\mathrm{Ru}(0001)$ as for example the catalytic oxidation of $\mathrm{CO} .{ }^{10}$ Recent experimental work ${ }^{12}$ shows that the $\mathrm{CO} / \mathrm{CO}_{2}$ conversion rate is still higher for oxygen coverages beyond $3 \mathrm{ML}$, which seems to imply that a high concentration of subsurface oxygen destabilizes the on-surface oxygen even further. The activity for $\mathrm{CO}$ oxi- dation on $\mathrm{Rh}(111)$ shows quite different behavior. ${ }^{13}$ It appears that when $\mathrm{Rh}$ is exposed to a high dose of oxygen the adsorption of $\mathrm{CO}$ and $\mathrm{CO}_{2}$ formation are poisoned due to surface oxide formation. ${ }^{14,15}$ Apparently, unreactive surface oxides are more likely to form on $\mathrm{Rh}$ than on Ru surfaces. Still, the microscopic process and mechanism of oxide formation on these transition-metal surfaces is not understood. Even the qualitative difference between a high-coverage oxygen adsorbate phase, e.g., a full on-surface monolayer plus subsurface oxygen, and a surface oxide is unclear; thus the detailed nature of these distinct states of oxygen has not been precisely defined yet. The present study was undertaken to specifically examine the structural and electronic properties of oxygen covered $\mathrm{Rh}(111)$ surfaces as a first step towards the understanding of the elementary steps governing the onset of surface oxide formation.

The surface crystal structure of oxygen covered $\mathrm{Rh}(111)$ has been studied experimentally using primarily low-energy electron diffraction (LEED) and scanning tunnel microscopy (STM). ${ }^{2,16-22}$ Only a few theoretical calculations have been devoted to the $\mathrm{O} / \mathrm{Rh}(111)$ system. So far, no fully selfconsistent calculations of the structural and electronic properties of $\mathrm{O}$ chemisorption on $\mathrm{Rh}(111)$ have been reported. Chen et al. ${ }^{23}$ investigated the bonding of atomic oxygen on $\mathrm{Rh}(111)$ using density-functional theory within the generalized gradient approximation (DFT-GGA) for an oxygen atom bonded to a ten-atom $\mathrm{Rh}$ cluster designed to model the (111) surface. Recently, Loffreda et $_{\text {al. }}{ }^{24}$ performed selfconsistent DFT-GGA calculations of the electronic properties of $\mathrm{O}$ on $\mathrm{Rh}(111)$, where the periodic system has been modeled by a two-dimensional rhodium slab with frozen geometry, on which $\mathrm{O}$ adlayers are set. Thus the fine details of the chemisorption structure have been neglected.

In this paper, we present results of density-functional calculations within the generalized gradient approximation for oxygen chemisorption on a seven-layer (111) Rh slab. Of particular interest was to determine the lowest-energy structures of the two lower coverage phases as reported from LEED intensity analyses, namely the $\mathrm{O}-(2 \times 2)(\Theta$ $=0.25 \mathrm{ML})$ and the $\mathrm{O}-(2 \times 1)(\Theta=0.5 \mathrm{ML})$ phases with 
oxygen adatoms at the fcc-hollow sites. ${ }^{19,20,25,26} \mathrm{We}$ also performed calculations for oxygen in the hcp-hollow sites. Higher coverage ordered structures, namely $\mathrm{O}-(1 \times 1) \quad(\Theta$ $=1 \mathrm{ML}$ ), have also been considered. For each coverage we performed a full structure optimization, including substrate relaxations. The results are in excellent agreement with the experimentally obtained atomic geometry for the $\mathrm{O}-(2 \times 2)$ and $\mathrm{O}-(2 \times 1)$ phases. We show that with increasing oxygen coverage the outermost substrate interlayer spacing expands considerably. In particular, we obtain that $1.0 \mathrm{ML}$ of $\mathrm{O}$ adatoms in a $(1 \times 1)$ structure at the fcc-hollow sites induces a relative expansion of $\sim 7 \%$ of the first $\mathrm{Rh}$ layer with respect to the bulk. This oxygen-induced effect on the $\mathrm{Rh}(111)$ substrate is much more pronounced than that reported for the neighboring $\mathrm{Ru}(0001)$ surface, ${ }^{10}$ for which $1 \mathrm{ML}$ of $\mathrm{O}$ causes a $3 \%$ relative expansion.

The stability of the $\mathrm{O}-(1 \times 1)$ adlayer structure on $\mathrm{Rh}(111)$ has been asserted by both Loffreda et al. and by the present calculations, which predict the adsorption of $\mathrm{O}$ in a $(1 \times 1)$ adlayer structure to be exothermic. Using molecular oxygen under UHV conditions the $(1 \times 1)$ phase has not been observed to form, and the saturation coverage for molecular oxygen exposure at room temperature remains a matter of controversy, ${ }^{21}$ with reported values ranging from 0.25 to $0.83 \mathrm{ML} \cdot{ }^{20,22,27,28}$ In this respect, the situation of $\mathrm{Rh}(111)$ resembles that of $\mathrm{Ru}(0001)$ before the work of Refs. 10 and 11 had appeared. The problem is that when dissociation of $\mathrm{O}_{2}$ is hindered by an energy barrier the experimental determination of the maximum atomic $\mathrm{O}$ surface coverage is very difficult. As was done for $\mathrm{Ru}(0001),{ }^{11}$ Schwegmann et al. tried to prepare an $\mathrm{O}-(1 \times 1)$ structure on $\mathrm{Rh}(111)$ by exposing $\mathrm{O}-(2 \times 1) / \mathrm{Rh}(111)$ to $\mathrm{NO}_{2}$ at $T=600 \mathrm{~K},{ }^{20}$ but in contrast to $\mathrm{Ru}$ they were not successful.

The remainder of this paper is organized as follows. In Sec. II, we specify the details of the first-principles calculations. In Sec. III, we present and discuss the results of the full structure optimization for the clean and O-covered $\mathrm{Rh}(111)$ surfaces and for the coverage dependence of the work function. In Sec. IV, we present results for the heat of dissociative adsorption of $\mathrm{O}_{2}$. The present results for $\mathrm{O}$ adsorbed on $\mathrm{Rh}(111)$ are discussed and compared with corresponding results for an $\mathrm{O}$-covered $\mathrm{Ru}(0001)$ surface that have appeared in the literature. In Sec. V, our results and conclusions are summarized.

\section{CALCULATIONAL DETAILS}

All the calculations have been performed using DFT and the GGA of Perdew et al. ${ }^{29}$ for the exchange-correlation functional as implemented in the all-electron full-potentiallinear augmented plane-wave method (FP-LAPW). ${ }^{30,31}$ This implementation includes total-energy and atomic force calculations, which allows a full structure optimization via a damped molecular-dynamics approach. ${ }^{32}$ The $\mathrm{Rh}(111)$ surface is modeled using the supercell approach, where we use a seven-layer (111) Rh slab with a vacuum region corresponding to six interlayer spacings. Oxygen atoms are adsorbed on both sides of the slab. Oxygen atoms and all atoms in the two outer Rh substrate layers were allowed to relax while the central three layers of the slab were fixed in their calculated bulk positions. For this calculation we used $R_{\mathrm{MT}}^{\mathrm{Rh}}$
$=2.3$ bohrs, $\quad E_{\text {cut }}=15.3 \mathrm{Ry}, \quad$ and a $(14 \times 14 \times 14)$ Monkhorst-Pack grid for the fcc bulk unit cell. The calculated bulk lattice constant changes by less than $0.1 \%$ if for these parameters values corresponding to those used for the surface calculations are used. The other LAPW parameters have the same values (see below) in both cases. In order to study the effect of oxygen adsorption on the electronic structure and properties of the substrate as a function of coverage $\Theta$, we have considered $\Theta=0.25,0.5$, and $1.0 \mathrm{ML} \mathrm{O}$ adlayers with a $(2 \times 2),(2 \times 1)$, and $(1 \times 1)$ periodicity. For a coverage of $0.25 \mathrm{ML}$, we optimized the structure maintaining the threefold rotation symmetry of the clean surface with $\mathrm{O}$ either in the fcc-hollow site or in the hcp-hollow site. For the $0.5 \mathrm{ML}$ case, the allowed symmetry of the adsorbate is reduced and oxygen atoms are also allowed to move laterally along the $[\overline{2} 11]$ direction. For the $1.0 \mathrm{ML}$ case, we considered $\mathrm{O}-(1 \times 1)$ adlayers with $\mathrm{O}$ atoms in the fcc- and hcphollow sites.

The FP-LAPW wave functions in the interstitial region are represented using a plane-wave expansion where the energy cutoff is taken to be $E_{\text {cut }}=16 \mathrm{Ry}$, and for the potential representation, plane waves up to $169 \mathrm{Ry}$ are considered. Inside the muffin-tin spheres $\left(R_{\mathrm{MT}}^{\mathrm{Rh}}=2.2\right.$ bohrs, $R_{\mathrm{MT}}^{\mathrm{O}}=1.4$ bohrs) the wave functions are expanded in spherical harmonics with $l$ up to 12 . A maximum of $l=6$ is considered for the wave functions entering in the evaluation of nonspherical matrix elements. For the expansion of the density and potential inside the spheres, a maximum of $l=4$ is used. The Brillouin-zone integration have been performed using a $(12 \times 12 \times 1)$ Monkhorst-Pack grid for the $(1 \times 1)$ surface unit cell. These are $180 \mathbf{k}$ points in the full surface Brillouin zone, which include the $\bar{\Gamma}$ point. For the $(2 \times 1)$ and $(2 \times 2)$ surface unit cells, grids have been chosen so as to obtain the same sampling of the reciprocal space. A temperature broadening with a Fermi function is used with a broadening parameter $T^{\mathrm{el}}=0.005 \mathrm{Ry}$ in order to reduce the number of $\mathbf{k}$ points that are necessary to calculate the total energy of the metallic systems. For the study of the $\mathrm{O}_{2}$ dimer, we have used different values for some of the LAPW parameters as indicated in the text below. Core states are treated fully relativistically, and for valence states, relativistic effects are included in a scalar relativistic treatment. The accuracy of the physical and numerical approximations made with the present calculational setup is discussed in the appendix.

\section{RESULTS AND DISCUSSION}

\section{A. Clean $\operatorname{Rh}(111)$}

In order to determine the effects of oxygen chemisorption on the $\mathrm{Rh}(111)$ surface structure, we first investigated the bare metal surface. The $\mathrm{Rh}$ substrate was set up with the calculated bulk lattice constant (DFT-GGA) of $a_{0}=3.83 \AA$, neglecting zero-point vibrations. The calculated cohesive energy is $E_{0}=6.1 \mathrm{eV}$ and the bulk modulus is $B$ $=2.59$ mbar. The experimental values for lattice constant, cohesive energy, and bulk modulus are $a_{0}=3.803 \AA, E_{0}$ $=5.75 \mathrm{eV}$, and $B=2.69$ mbar, respectively. ${ }^{33,34}$ The calculated values are in line with other DFT-GGA results. ${ }^{35,36}$ For the clean $\mathrm{Rh}(111)$ surface a contraction of the two outer interlayer spacings with respect to the bulk value $d_{0}$ by 

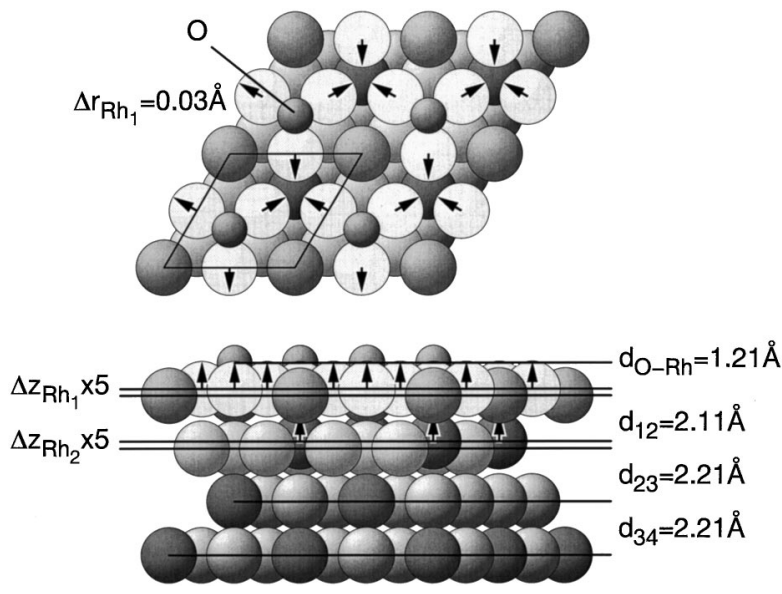

FIG. 1. Top and side view of the $\mathrm{O}-(2 \times 2) / \mathrm{Rh}(111)$ structure. The arrows indicate the direction of the atomic displacements of the substrate atoms $\Delta r_{\mathrm{Rh}_{1}}, \Delta z_{\mathrm{Rh}_{1}}$, and $\Delta z_{\mathrm{Rh}_{2}}$ of Table I. Small dark grey circles represent oxygen atoms, and large white and grey circles represent $\mathrm{Rh}$ atoms, where those lying in the same plane and equivalent under the threefold rotation symmetry have the same color.

$\Delta_{12} / d_{0}=-1.8 \%$ and $\Delta_{23} / d_{0}=-0.9 \%$ is obtained. These values are in good agreement with the surface relaxations for $\mathrm{Rh}(111)$ determined by recent LEED analyses; in Ref. 37, the values $\Delta_{12} / d_{0}=(-1.4 \pm 0.9) \%$ and $\Delta_{23} / d_{0}=(-1.4$ $\pm 1.8) \%$ and, in Ref. 20, $\Delta_{12} / d_{0}=(-0.9 \pm 0.9) \%$ and $\Delta_{23} / d_{0}=(-0.0 \pm 0.9) \%$, have been reported. Other DFT calculations, however, using the local-density approximation (LDA) reported $\Delta_{12} / d_{0}=-2.5 \%$ (Ref. 38) employing the full-potential-linear muffin-tin orbital method, and $\Delta_{12} / d_{0}$ $=(-1.7 \pm 0.2) \%, \Delta_{23} / d_{0}=(-0.25 \pm 0.15) \%$, and $\Delta_{34} / d_{0}$ $=(+0.60 \pm 0.15) \%$ using ultrasoft pseudopotentials. ${ }^{39}$

\section{B. Coverage dependent $O$-induced surface relaxations}

The chemisorption of $\mathrm{O}$ atoms on a $\mathrm{Rh}(111)$ surface was investigated at three coverages, $\Theta=0.25,0.5$, and $1.0 \mathrm{ML}$ with oxygen occupying either fcc or hcp sites. We present here the results of the structure optimization for the different coverages of $\mathrm{O}$ chemisorption on the fcc site and compare them with available results of LEED analyses. The coverage dependence of the oxygen-induced $\mathrm{Rh}$ surface relaxations will also be discussed.

The calculated atomic geometries of the $\mathrm{O}-(2 \times 2)$ and $\mathrm{O}-(2 \times 1)$ covered $\mathrm{Rh}(111)$ surfaces are displayed in Figs. 1 and 2 and compared with the results of dynamical LEED analyses in Tables I and II, respectively. At $\Theta=0.25 \mathrm{ML}$, Schwegmann et al. ${ }^{20}$ performed a LEED analysis optimizing the oxygen-rhodium interlayer spacing $d_{\mathrm{O}-\mathrm{Rh}}$, as well as lateral and vertical displacements of $\mathrm{Rh}$ atoms in the outermost $\mathrm{Rh}$ layer while preserving the local threefold rotational symmetry of the adsorbate. In the present calculations, atoms of the second substrate layer were also allowed to relax. At the stable adsorption geometry the triangle formed by the three $\mathrm{Rh}$ atoms in the first layer below the $\mathrm{O}$ atom has laterally expanded. The calculated amplitude of these in-plane displacements, $\Delta r_{\mathrm{Rh}_{1}}$, radially away from the chemisorbed oxygen as indicated in Fig. 1, is $0.03 \AA$, in good agreement with the results of the LEED analysis of Ref. 20. The fully relaxed

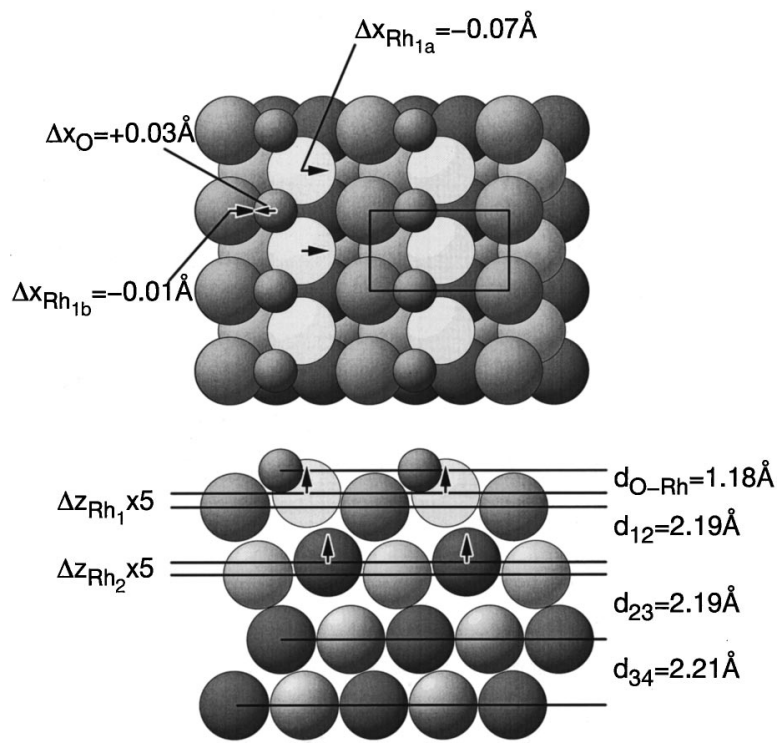

FIG. 2. Top and side view of the $\mathrm{O}-(2 \times 1) / \mathrm{Rh}(111)$ structure. The arrows indicate the direction of reference for the atomic inplane displacements $\Delta x_{\mathrm{O}}, \Delta x_{\mathrm{Rh}_{1 a}}$, and $\Delta x_{\mathrm{Rh}_{1 b}}$ and for the vertical displacements $\Delta z_{\mathrm{Rh}_{1}}$ and $\Delta z_{\mathrm{Rh}_{2}}$ of Table II. Small dark grey circles represent oxygen atoms, and large white and grey circles represent $\mathrm{Rh}$ atoms, where those lying in the same plane and symmetry equivalent have the same color.

$\mathrm{O}-\mathrm{Rh}$ bond length for adsorption on the fcc-hollow site is $2.00 \AA$, with oxygen adatoms $1.21 \AA$ above the closest $\mathrm{Rh}$ layer (see Fig. 1). Moreover, 0.25 ML of oxygen induces a small buckling, $\Delta z_{\mathrm{Rh}}$, in the two outermost $\mathrm{Rh}$ layers of about $0.13 \AA$ and $0.08 \AA$, respectively. Calculated vertical spacings are indicated in Fig. 1 and in Table I mean interlayer spacings are compared with the results of the LEED data analysis of Ref. 20. The high quality of the DFT-GGA atomic geometry is clearly manifested by the results listed in Table I. Both the results of the present calculations and those of the LEED analysis ${ }^{20}$ agree that the mean outermost substrate interlayer spacing $\bar{d}_{12}$ is expanded relative to the corresponding value for the clean surface. We have calculated an increase from $2.17 \AA$ to $2.25 \AA$ compared to the resulting

TABLE I. Calculated structural parameters in $\AA$ for the $\mathrm{O}-(2 \times 2) / \mathrm{Rh}(111)$ structure with $\mathrm{O}$ in the fcc-hollow position. The experimental data are from Ref. 20. The in-plane displacements of the first substrate layer atoms (see Fig. 1) are denoted as $\Delta r_{\mathrm{Rh}_{1}}$. For the interlayer distances, the center of mass of each Rh layer is used. Numbers in parentheses correspond to bulk values, which were fixed. O-Rh indicates the bond length and $\Delta z_{\mathrm{Rh}_{1}}, \Delta z_{\mathrm{Rh}_{2}}$ the buckling in the first and second outermost layers, respectively.

\begin{tabular}{lcc}
\hline \hline & DFT-GGA & Ref. 20 \\
\hline O-Rh & 2.00 & $2.00 \pm 0.08$ \\
$\bar{d}_{\mathrm{O}-\mathrm{Rh}}$ & 1.24 & $1.24 \pm 0.06$ \\
$\bar{d}_{12}$ & 2.25 & $2.24 \pm 0.04$ \\
$\bar{d}_{23}$ & 2.22 & $(2.194)$ \\
$\bar{d}_{34}$ & $(2.213)$ & \\
$\Delta z_{\mathrm{Rh}_{1}}$ & 0.13 & $0.06 \pm 0.05$ \\
$\Delta z_{\mathrm{Rh}_{2}}$ & 0.06 & \\
\hline \hline
\end{tabular}


TABLE II. Calculated structural parameters in $\AA$ for the $\mathrm{O}-(2 \times 1) / \mathrm{Rh}(111)$ structure. The experimental data are from Refs. 20 and 19. The lateral displacements of the atoms $\mathrm{Rh}_{1 a}$ and $\mathrm{Rh}_{1 b}$ (see Fig. 2) are denoted as $\Delta x_{\mathrm{Rh}_{\mathrm{i}}}$, and for the interlayer distances, the center of mass of each Rh layer is used. Numbers in parentheses correspond to bulk values, which were fixed. $\mathrm{O}-\mathrm{Rh}_{i}$ indicate bond lengths and $\Delta z_{\mathrm{Rh}_{1}}, \Delta z_{\mathrm{Rh}_{2}}$ the buckling in the first and second outermost layers, respectively.

\begin{tabular}{lccc}
\hline \hline & DFT-GGA & Ref. 20 & Ref. 19 \\
\hline${\mathrm{O}-\mathrm{Rh}_{1 a}}$ & 2.00 & $2.02 \pm 0.08$ & $2.01 \pm 0.08$ \\
$\mathrm{O}-\mathrm{Rh}_{1 b}$ & 1.98 & $2.00 \pm 0.08$ & $1.92 \pm 0.08$ \\
$\bar{d}_{\mathrm{O}-\mathrm{Rh}}$ & 1.23 & $1.25 \pm 0.05$ & 1.22 \\
$\bar{d}_{12}$ & 2.28 & $2.26 \pm 0.04$ & 2.23 \\
$\bar{d}_{23}$ & 2.23 & $(2.194)$ & 2.21 \\
$\bar{d}_{34}$ & $(2.213)$ & & $(2.20)$ \\
$\Delta z_{\mathrm{Rh}_{1}}$ & 0.09 & $0.04 \pm 0.04$ & $0.07 \pm 0.03$ \\
$\Delta z_{\mathrm{Rh}_{2}}$ & 0.08 & & $0.03 \pm 0.03$ \\
$\Delta x_{\mathrm{O}}$ & +0.03 & $+0.00 \pm 0.08$ & $+0.05 \pm 0.08$ \\
$\Delta x_{\mathrm{Rh}_{1 a}}$ & -0.07 & $-0.09 \pm 0.10$ & -0.04 \\
$\Delta x_{\mathrm{Rh}_{1 b}}$ & -0.01 & $-0.01 \pm 0.10$ & -0.05 \\
\hline \hline
\end{tabular}

increase of $0.06 \AA$ (from $2.174 \AA$ to $2.235 \AA$ ) of the LEED analysis. ${ }^{20}$ The calculated and LEED determined interlayer spacings of the bulk-terminated surface are $2.21 \AA$ and $2.194 \AA$, respectively, which means that the contraction of the outermost $\mathrm{Rh}$ interlayer spacing has been completely removed by oxygen adsorption at a quarter of a monolayer.

For the higher coverage $\mathrm{O}-(2 \times 1)$ structure, we also only present the optimized structure with oxygen adatoms in the fcc-hollow sites. As for the $\Theta=0.25 \mathrm{ML}$ coverage, Rh atoms of the second substrate layer are allowed to relax. The resulting atomic structure with the unit cell used to describe the $0.5 \mathrm{ML}$ coverage is shown in Fig. 2. The structural characteristics are the following: The $\mathrm{O}$ atoms are adsorbed slightly displaced, $\Delta x_{\mathrm{O}}=0.03 \AA$, from the center of the fcc site as indicated in Fig. 2. Simultaneously, small lateral displacements of the bulk-terminated $\mathrm{Rh}(111)$ surface $\Delta x_{\mathrm{Rh}}$ in the outer substrate layer of up to $0.07 \AA$ in the opposite direction were induced by the adsorption. The in-plane forces exerted on the second substrate $\mathrm{Rh}$ atoms are zero at the minimumenergy position. The vertical spacings are also indicated in Fig. 2. Both the two outer Rh layers are slightly buckled. In Table II, where we compare the DFT-GGA atomic geometry with the results of the LEED analyses of Ref. 20 and Ref. 19 for the interlayer distances, the center of mass of each layer is used. O-Rh bond lengths are also given. In Ref. 20 only vertical and lateral displacements of the $\mathrm{O}$ layer and the outermost $\mathrm{Rh}$ layer were considered in the analysis. The good quality of the DFT-GGA atomic geometry for this higher coverage becomes evident by inspection of Table II. The DFT-GGA and LEED in-plane relaxations of the Rh atoms of the outer substrate layer and the lateral displacement of the $\mathrm{O}$ atoms agree to within the experimental uncertainties. The agreement is also good for the O-Rh bond lengths, for the height of $\mathrm{O}$ atoms above the outermost $\mathrm{Rh}$ plane, and for the buckling of the atoms of the surface layer. For this higher-coverage structure, the calculated mean outer substrate interlayer spacing $\bar{d}_{12}$ shows a larger expansion with
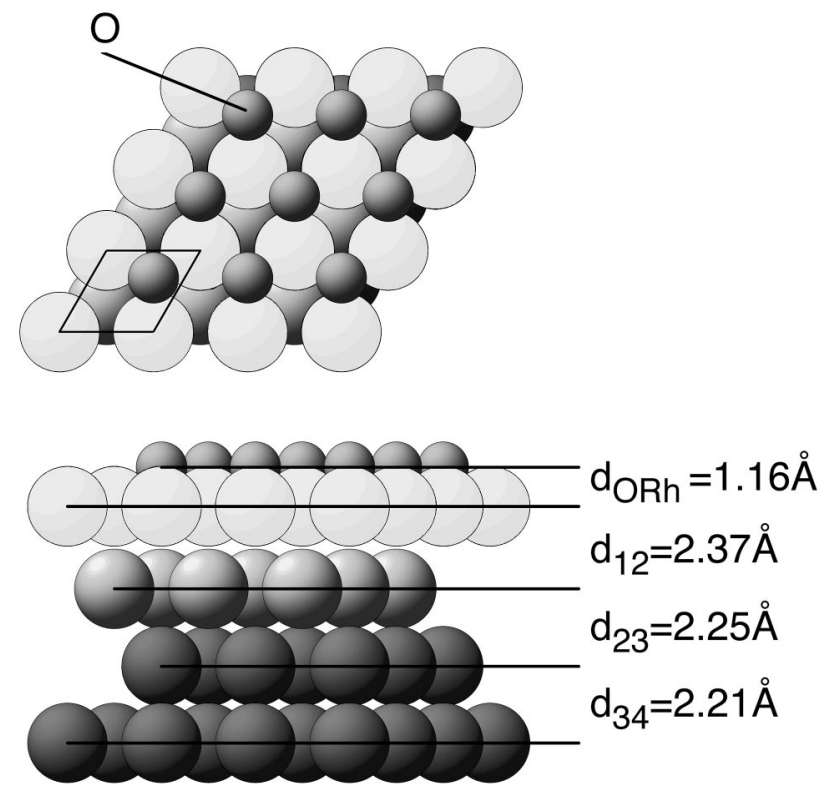

FIG. 3. Top and side view of the $\mathrm{O}-(1 \times 1) / \mathrm{Rh}(111)$ structure. Small dark grey circles represent oxygen atoms, and large white and grey circles represent $\mathrm{Rh}$ atoms, where those lying in the same plane and symmetry equivalent have the same color.

respect to the relaxed clean surface than the lower coverage $\Theta=0.25 \mathrm{ML}$ structure, from $2.17 \AA$ to $2.28 \AA$. In Ref. 20 , an increase in $\bar{d}_{12}$ from $2.174 \AA$ to $2.260 \AA$ has been reported.

For the highest coverage of $\Theta=1.0 \mathrm{ML}$, unlike the lower-coverage cases, no in-plane relaxation is allowed in the calculations due to the symmetry of the unit cell. The resultant top and side views with $\mathrm{O}$ in the fcc-hollow site are shown in Fig. 3 and listed in Table III. We have calculated an outermost interlayer spacing of $2.37 \AA$.

Upon $\mathrm{O}$ chemisorption the changes in the mean outermost $\mathrm{Rh}$ interlayer spacing $\Delta d_{12}$ relative to the unrelaxed clean surface interlayer spacing $d_{0}$ strongly vary with coverage. The results are summarized in Fig. 4. The clean $\mathrm{Rh}(111)$ surface is contracted by $1.8 \%$, but only very small amounts of chemisorbed oxygen $(\sim 0.1 \mathrm{ML})$ are needed to remove that contraction. Coverages of $\Theta=0.25,0.50$, and $1.0 \mathrm{ML}$ induce relative expansions $\Delta d_{12} / d_{0}$ of $1.5 \%, 3.0 \%$, and $7.2 \%$, respectively. LEED intensity analysis ${ }^{20}$ yields a contracted clean $\mathrm{Rh}(111)$ surface $(0.9 \%)$ and an expanded surface layer upon adsorption for $\Theta=0.25 \mathrm{ML}$ by $1.9 \%$ and for $\Theta=0.50 \mathrm{ML}$ by $3.0 \%$ with respect to the bulk value.

TABLE III. Calculated structural parameters in $\AA$ for the $\mathrm{O}-(1 \times 1) / \mathrm{Rh}(111)$ structure with $\mathrm{O}$ adatoms on fcc sites. Numbers in parentheses correspond to bulk values, which were fixed. O-Rh indicates bond length.

\begin{tabular}{lc}
\hline \hline & DFT-GGA \\
\hline O-Rh & 1.95 \\
$\bar{d}_{\mathrm{O}-\mathrm{Rh}}$ & 1.16 \\
$\bar{d}_{12}$ & 2.37 \\
$\bar{d}_{23}$ & 2.25 \\
$\bar{d}_{34}$ & $(2.213)$ \\
\hline \hline
\end{tabular}




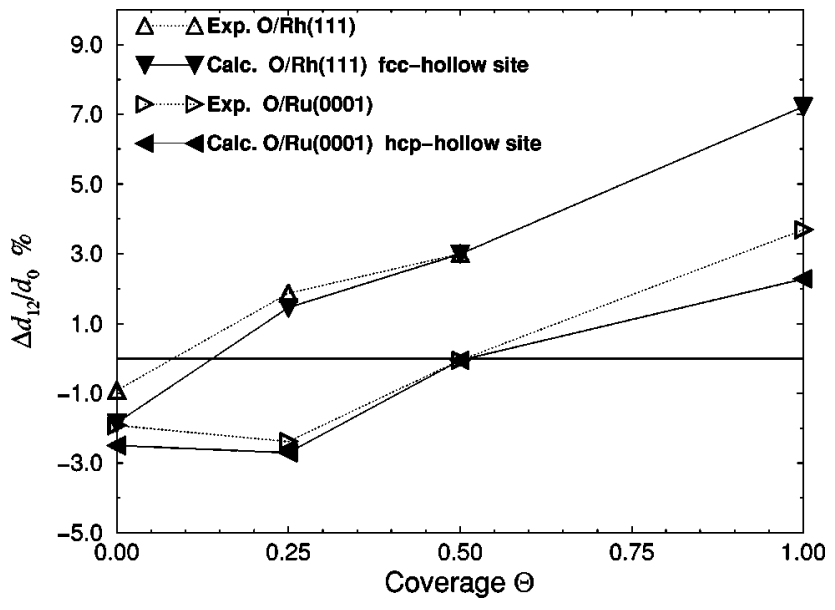

FIG. 4. Calculated change in the mean outermost interlayer spacing relative to that of the bulk-terminated surface as a function of the oxygen coverage for the $\mathrm{O} / \mathrm{Rh}(111)$ system with $\mathrm{O}$ in the fcc-hollow sites. Calculated values for $\mathrm{O} / \mathrm{Ru}(0001)$ with $\mathrm{O}$ in the hcp-hollow sites are those of Ref. 10. Experimental values for the $\mathrm{O} / \mathrm{Rh}(111)$ (Ref. 20) and $\mathrm{O} / \mathrm{Ru}(0001)$ (Ref. 20) systems are also given.

It is interesting to compare these results for $\mathrm{O} / \mathrm{Rh}(111)$ to those of $\mathrm{O} / \mathrm{Ru}(0001)$. Prior LEED work on the $\mathrm{O} / \mathrm{Ru}(0001)$ system $^{20,40}$ and DFT calculations ${ }^{10}$ show that oxygen chemisorption also causes a gradual removal of the clean $\mathrm{Ru}(0001)$ surface contraction, but the effect is much less pronounced than for Rh(111). The DFT calculated oxygen-induced relative changes in the outer interlayer spacing of $\mathrm{Ru}(0001)$ from low coverages up to a full monolayer, where the $\mathrm{O}$ adatoms occupy hcp-hollow sites, ${ }^{10}$ and the corresponding results of the LEED analysis of Ref. 20 are displayed in Fig. 4. We observe that a coverage of $\Theta=0.5 \mathrm{ML}$ on $\mathrm{Rh}(111)$ induces a similar relative expansion of the outer interlayer spacing as the higher coverage of $\Theta=1.0 \mathrm{ML}$ does on $\mathrm{Ru}(0001)$ and that $\Theta \sim 0.5 \mathrm{ML}$ is needed to remove the contraction of the clean $\mathrm{Ru}(0001)$ surface, in contrast to the already mentioned $\Theta \sim 0.1 \mathrm{ML}$ needed for $\mathrm{Rh}(111)$.

These results can be interpreted on the basis of rather simple ideas. The outermost atomic layer of most clean transition-metal surfaces shows an inward relaxation. As explained by Methfessel et al., ${ }^{38}$ the magnitude of this relaxation shows a roughly parabolic dependence with $d$ occupation across the series, being largest for a half-full band. This trend is related to the well-known parabolic dependence on the $d$ occupation of the cohesive and surface energies, which are roughly proportional to the square root of the energy per nearest-neighbor bond. Experimental values for the cohesive energy of the $\mathrm{Ru}$ and $\mathrm{Rh}$ bulk are $E_{\mathrm{coh}}^{\mathrm{Ru}}=6.74 \mathrm{eV} /$ atom and $E_{\mathrm{coh}}^{\mathrm{Rh}}=5.75 \mathrm{eV} /$ atom. ${ }^{34}$ Cutting some of the nearest-neighbor bonds on an atom to create the surface enhances the strength of the remaining ones, which tends to contract the surface and gives rise to tensile surface stress. The strength of the bonds at $\mathrm{Ru}(0001)$ is stronger than those at $\mathrm{Rh}(111)$. Thus the inward relaxation of the clean $\mathrm{Ru}(0001)$ surface is larger than that of $\mathrm{Rh}(111)$. It is actually the combination of this effect with that of the $s p$-electrons spillout that governs the relaxation trend across the series. ${ }^{38}$

Upon oxygen adsorption on $\mathrm{Rh}(111)$ electron density is reduced between the two outermost substrate layers, which
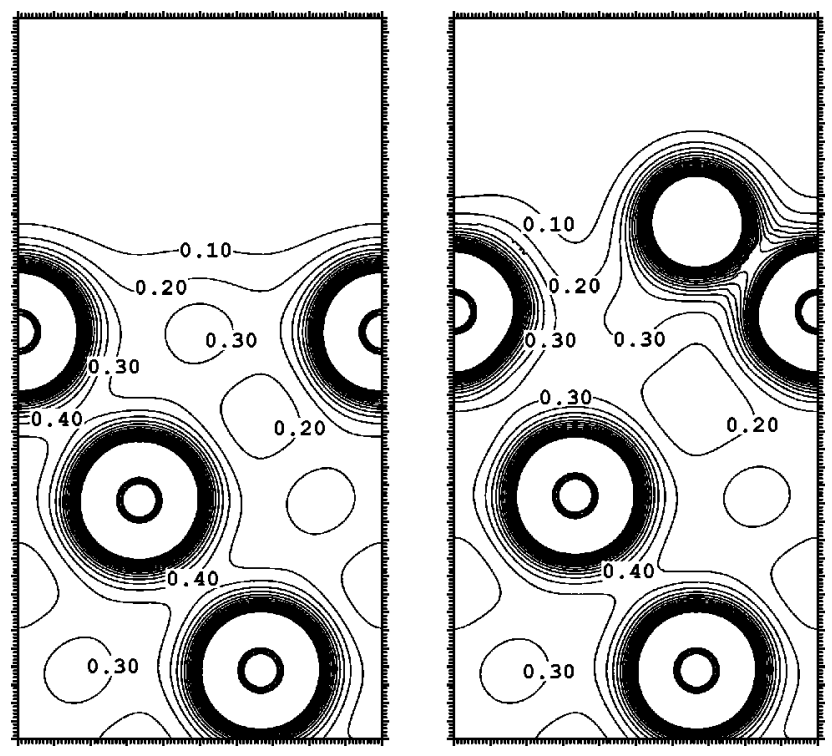

FIG. 5. Contour plots of constant electron density in a [ $\overline{2} 11]$ plane perpendicular to the (111) surface of $\mathrm{Rh}$ without (left) and with a $1 \mathrm{ML}$ of atomic $\mathrm{O}$ adsorbed in a $(1 \times 1)$ structure on fcchollow sites (right). Distances between constant-density contours is $0.1 \mathrm{e} / \AA^{3}$. Notice that $\mathrm{O}$ adsorption removes bonding charge from the bond between the $\mathrm{Rh}$ atoms, which lie directly under the $\mathrm{O}$ adatoms in the first substrate layer and their nearest-neighbors in the second substrate layer. The distances between each $\mathrm{Rh}$ atom that is adjacent to an $\mathrm{O}$ and its subsurface $\mathrm{Rh}$ nearest-neighbors exceeds the bulk Rh nearest-neighbor separation $R_{n n}$ by $4.9 \%$. Bond lengths between neighboring $\mathrm{Rh}$ atoms on the second and third substrate layers is $1.2 \%$ longer than $R_{n n}$. Corresponding bond lengths for the clean $\mathrm{Rh}(111)$ surface are $1.2 \%$ and $0.6 \%$ shorter than $R_{n n}$, respectively.

empties $\mathrm{Rh}-\mathrm{Rh}$ bonding states. Thus, the $\mathrm{Rh}-\mathrm{Rh}$ bonds get weakened giving rise to the oxygen-induced increase of the outermost interlayer spacing of the Rh substrate at all coverages. Figure 5 illustrates it for one example by showing contour plots of constant electron density in a [ $\overline{2} 11]$ plane perpendicular to the clean $\mathrm{Rh}(111)$ (left) and the $\mathrm{O}-(1 \times 1) / \mathrm{Rh}(111)$ (right) surfaces with $\mathrm{O}$ adatoms in fcchollow sites. Figure 5 clearly shows that $\mathrm{O}$ adsorption removes bonding charge from the bond between the $\mathrm{Rh}$ atoms, which lie directly under the $\mathrm{O}$ adatoms in the first substrate layer and their nearest neighbors in the second substrate layer. Note that the charge density has been reduced from 0.4 $e / \AA^{3}$ (between neighboring $\mathrm{Rh}$ atoms on the second and third substrate layers) to $0.3 \mathrm{e} / \AA^{3}$. Thus, Fig. 5 confirms that the interaction between the negative $\mathrm{O}$ and its first $\mathrm{Rh}$ neighbors works against the bonding of the latter to second-layer $\mathrm{Rh}$ atoms. This adsorbate-induced effect is stronger on $\mathrm{Rh}(111)$ than on $\mathrm{Ru}(0001)$ because surface bonds at the clean $\mathrm{Rh}(111)$ surface are weaker as discussed above.

In all the order phases of $\mathrm{O}$ on $\mathrm{Rh}(111)$ that form in nature, $\mathrm{O}$ adsorbs in the fcc site and on $\mathrm{Ru}(0001)$ in the hcp site. These are the sites that a next substrate layer would occupy; they are stabilized by a lowering of the occupied density of states (DOS), following the trend that systems like to attain a chemically-hard electronic structure, i.e., a low density at the Fermi level. Thus essentially the same effect that stabilizes the fcc structure of bulk Rh and hcp of bulk 

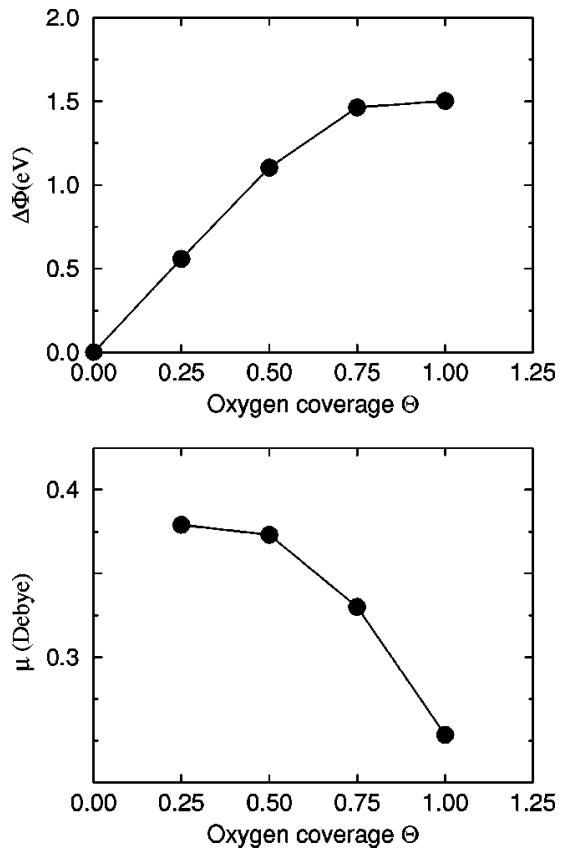

FIG. 6. Calculated work-function change (top) and adatom dipole moment (bottom) for $\mathrm{O}$ on $\mathrm{Rh}(111)$ as a function of the oxygen coverage $\Theta$. Oxygen atoms occupy the fcc-hollow sites.

$\mathrm{Ru} .{ }^{41}$ We shall discuss in more detail the preferred fcc adsorption site for $\mathrm{O}$ on $\mathrm{Rh}(111)$ in Sec. IV.

\section{Coverage dependence of the work function}

To gain insight into the nature of the O-Rh bond, we discuss the work-function change as a function of coverage. The calculated work function of the clean $\mathrm{Rh}(111)$ surface is $\Phi=5.23 \mathrm{eV}$. Experimental values are $4.98 \mathrm{eV}$ for polycrystalline $\mathrm{Rh}$ (Ref. 42) and $5.3 \mathrm{eV}$ for crystalline $\mathrm{Rh}(111){ }^{43}$ Previous DFT-LDA studies ${ }^{38}$ calculated the value $\Phi$ $=5.44 \mathrm{eV}$. Figure 6 shows the calculated work-function change $\Delta \Phi$ as a function of oxygen coverage $\Theta$. Initially, the work function rises linearly as a function of $\Theta$; at $\Theta$ $=0.75 \mathrm{ML}$ it reaches its saturation value. As can be noted, we also performed calculations for a structure with coverage $\Theta=0.75$ ML. In this structure $\mathrm{O}$ atoms are placed in fcchollow sites in the $(2 \times 2)$ surface unit cell. Such an increase in the work function upon oxygen adsorption reflects the high electronegativity of oxygen that results in an induced inward dipole moment, i.e., with the negative charge at the vacuum side of the surface. This is clearly seen in Fig. 7, which shows contours of constant charge density, in a [211] plane perpendicular to the (111) surface, of the charge-density difference between the chemisorbed system [O- $(2 \times 2)$ with $\mathrm{O}$ in fcc-hollow sites] and the sum of both the charge density of the clean $\mathrm{Rh}(111)$ surface (with distances between $\mathrm{Rh}$ atoms as in the chemisorbed system) and that of the isolated adatom. $\Delta \Phi(\Theta)$ is the electrostatic field built by the adsorbate-induced dipole moment $\mu(\Theta)$, and Fig. 6 shows how $\Delta \Phi$ and $\mu$ change with increasing coverage. The coverage dependence of the induced dipole per adatom is a consequence of the dipole-dipole interaction giving rise to a depolarization with decreasing $\mathrm{O}-\mathrm{O}$ distance. At lower coverages $\mathrm{O}$ adatoms are partially negatively charged

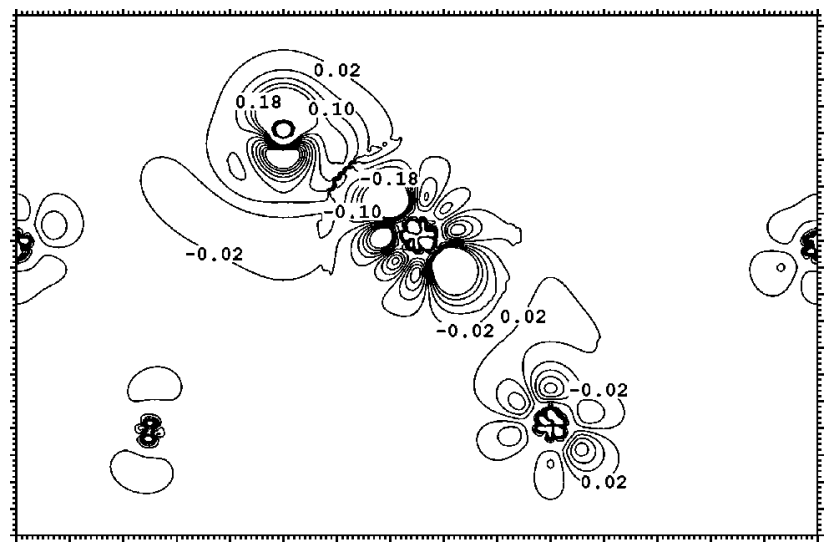

FIG. 7. Contours of constant charge density in a [ $\overline{2} 11]$ plane perpendicular to the (111) surface of $\mathrm{O} / \mathrm{Rh}$ with $0.25 \mathrm{ML}$ of atomic $\mathrm{O}$ adsorbed in a $(2 \times 2)$ structure in fcc-hollow sites. Contours correspond to a charge-density difference, namely, that of the chemisorbed system from which both the charge density of the clean $\mathrm{Rh}(111)$ surface (with distances between $\mathrm{Rh}$ atoms as in the chemisorbed system) and that of the isolated adatom layer has been subtracted. Distances between constant-density contours is $0.04 \mathrm{e} / \AA^{3}$. The picture displays the induced effect by the adsorption of a high electronegative $\mathrm{O}$ atom on the valence charge of its nearestneighbor metal atoms.

implying a significant adsorbate-adsorbate repulsion. Reducing the electron transfer and the oxygen-induced dipole is energetically favorable for higher coverages as it reduces the $\mathrm{O}-\mathrm{O}$ repulsion.

\section{HEAT OF ADSORPTION OF $\mathrm{O}_{2}$}

An estimate of the stability of $\mathrm{O}$ adlayer structures on $\mathrm{Rh}(111)$ with respect to adsorption of $\mathrm{O}_{2}$ is of considerable interest because dissociative adsorption of $\mathrm{O}_{2}$ molecules represents an important step in many catalytic reactions. The average binding energy per oxygen atom as a function of the coverage $\Theta$ is

$$
E_{b}(\Theta)=-\frac{1}{N_{0}}\left[E_{\mathrm{O}, \mathrm{Rh}}(\Theta)-\left(E_{\mathrm{Rh}}+N_{0} E_{\mathrm{O}}\right)\right],
$$

where $N_{0}$ is the number of oxygen atoms in the unit cell, $E_{\mathrm{O}, \mathrm{Rh}}, E_{\mathrm{Rh}}$, and $E_{\mathrm{O}}$ represent the total energies per unit cell of the Rh slab with oxygen adatoms, the clean Rh slab, and the free oxygen atom, respectively. The heat of adsorption of $\mathrm{O}_{2}$ is then

$$
\Delta H_{\mathrm{ad}}(\Theta)=2 E_{b}(\Theta)-D,
$$

where $D$ is the dissociation energy of the $\mathrm{O}_{2}$ molecule. A positive value for the heat of adsorption indicates that the dissociative adsorption of $\mathrm{O}_{2}$ is exothermic. That is, the binding energy per $\mathrm{O}$ adatom on $\mathrm{Rh}(111)$ is larger than that which the $\mathrm{O}$ atoms have in $\mathrm{O}_{2}$ (gas), i.e., $D / 2$. Binding energy $E_{b}$ and adsorption energy per $\mathrm{O}$ atom $E_{\text {ad }}=\Delta H_{\mathrm{ad}} / 2$ differ by half of the dissociation energy of the $\mathrm{O}_{2}$ molecule.

The calculations of the total energy of an isolated $\mathrm{O}$ atom, which are involved in the calculation of the atomic oxygen binding energies [see Eq. (1)], have been performed using the local spin-density approximation and the generalized gra- 
TABLE IV. Adsorption energies, $E_{\mathrm{ad}}=\Delta H_{\mathrm{ad}} / 2$ [Eq. (2)], in eV/ atom for the adlayers structures investigated.

\begin{tabular}{cccc}
\hline \hline Structure & Coverage & fcc-hollow site & hcp-hollow site \\
\hline O- $(2 \times 2)$ & 0.25 & 2.24 & 2.13 \\
O- $(2 \times 1)$ & 0.5 & 1.95 & 1.81 \\
O- $(1 \times 1)$ & 1 & 1.40 & 1.22 \\
\hline \hline
\end{tabular}

dient approximation in a cubic cell of side length 15 bohrs with $\Gamma$-point sampling of the Brillouin zone (other parameters as in Sec. II) without spherically averaging the electron density in the open shell. For the calculation of the dissociation energy of the isolated $\mathrm{O}_{2}$ molecule, $D$ total energies of $\mathrm{O}_{2}$ and atomic $\mathrm{O}$ have been determined using the generalized gradient approximation in a cubic cell of side length 15 bohrs with $\Gamma$-point sampling of the Brillouin zone, $E_{\text {cut }}$ $=24 \mathrm{Ry}$, and $R_{\mathrm{MT}}^{\mathrm{O}}=1.1$ bohrs (other parameters as in Sec. II); the electron density in the $\mathrm{O}$ atom open shell is not spherically averaged. Here, the oxygen muffin-tin radius is smaller compared to that used for the study of the chemisorbed systems. Therefore, the kinetic-energy cutoff for the plane-wave basis needed for the interstitial region is larger. The binding energy per $\mathrm{O}$ atom in $\mathrm{O}_{2}$ is calculated to be $D / 2=2.983 \mathrm{eV} /$ atom and the bond distance 2.3011 bohrs.

The experimental results for the $\mathrm{O}_{2}$ binding energy and for the ground-state bond distance are $2.560 \mathrm{eV} /$ atom per $\mathrm{O}$ atom $^{44}$ and 2.2818 bohrs, respectively. ${ }^{45}$ The overestimation of binding energies and bond distances are in line with earlier density-functional calculations that included gradient corrections. ${ }^{46-50}$

Table IV and Fig. 8 summarize the calculated $\mathrm{O}$ adsorption energies, $E_{\mathrm{ad}}$ of $\mathrm{O}$ on $\mathrm{Rh}(111)$ for the surface structures investigated. Binding energies for adsorption of oxygen on
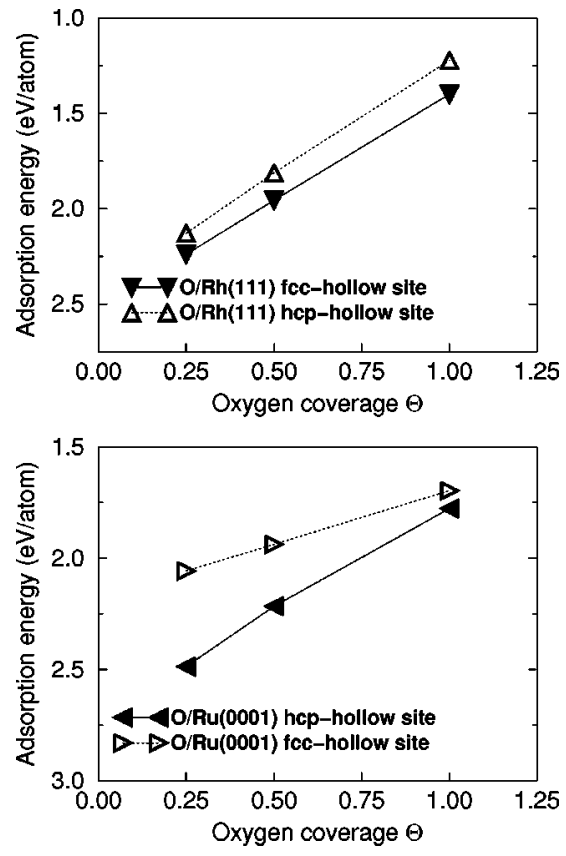

FIG. 8. Calculated adsorption energy (top), $E_{\text {ad }}=\Delta H_{\text {ad }}(\Theta) / 2$ in eV/atom, as a function of oxygen coverage $\Theta$ on $\mathrm{Rh}(111)$ for the surface structures investigated. Calculated values for $\mathrm{O} / \mathrm{Ru}(0001)$ (bottom) are from Ref. 10.

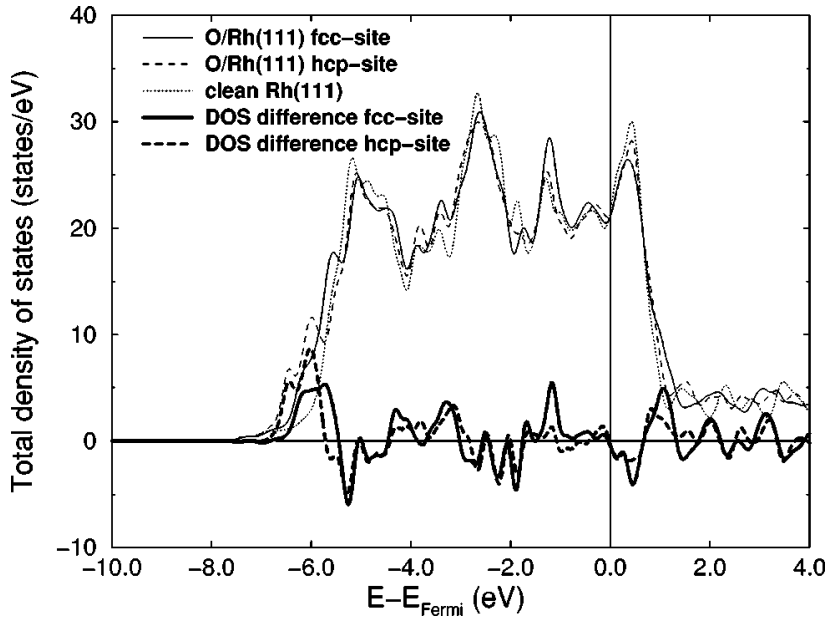

FIG. 9. Calculated total densities of states (states/eV) for the $\mathrm{O}-(2 \times 2) / \mathrm{Rh}(111)$ structures considered and for the clean $\mathrm{Rh}(111)$ surface. Thicker lines show total DOS differences for $\mathrm{O}$ adatoms in the fcc (solid line) and in the hcp (dashed line) hollows.

$\mathrm{Rh}(111)$ in fcc- as compared to hcp-hollow sites differ by less than $0.2 \mathrm{eV} / \mathrm{atom}$. This difference in binding between fcc and hep hollows remains relatively constant and small as a function of coverage but clearly favors the fcc sites in agreement with the results of LEED analyses ${ }^{19,20}$ and ion scattering data. ${ }^{25}$ Because the difference in binding energy between the fcc- and hcp-hollow sites is small, one may expect that a mixed site occupation would be preferred at finite temperatures. It is interesting to compare these results with those for $\mathrm{O}$ adsorption on $\mathrm{Ru}(0001)$. Figure 8 shows the DFT-GGA calculated adsorption energies of $\mathrm{O}$ on $\mathrm{Ru}(0001)$ using pseudopotentials of Ref. 10. We have already mentioned that $\mathrm{O}$ on $\mathrm{Ru}(0001)$ occupies hcp-hollow sites, i.e., the sites that a next substrate layer would occupy. We note that at lower coverages the hcp-hollow sites are favored over the fcc-hollow sites by as much as $0.4 \mathrm{eV} /$ atom.

In the following we will analyze in simple terms, i.e., qualitatively, the stronger $\mathrm{O}$ binding in fcc sites on $\mathrm{Rh}(111)$. The leading structural differences at the two kinds of hollows is the location of atoms in the second-neighbor shell; thus a small difference in adatom binding energies at the two sites is to be expected. The scenario that leads to the fcc-site preference begins with the observation that the bond between $\mathrm{O}$ and $\mathrm{Rh}$ atoms is not purely covalent. We argue that the $\mathrm{O}$ site preference on $\mathrm{Rh}(111)$ is based on a stronger ionic bonding in the fcc site and that the covalent bonding between $\mathrm{O}$ and $\mathrm{Rh}$ substrate atoms for both hollow sites is virtually identical.

Figure 9 shows the total density of states of the $\mathrm{O}-(2 \times 2) / \mathrm{Rh}(111)(\Theta=0.25 \mathrm{ML})$ slabs, with $\mathrm{O}$ at the fccand hcp-hollow sites, and of the clean $\mathrm{Rh}(111)$ slab. The differences between the O-covered and the clean $\mathrm{Rh}(111)$ total DOS, which give the $\mathrm{O}$ adatom total DOS, are little affected by the location of the adatom on the surface. That is, Fig. 9 reflects that the covalent bonding between an $\mathrm{O}$ adatom and its $\mathrm{Rh}$ neighbors at both sites is practically identical. A telling feature of the computed electronic structure properties are the work functions and the relative positions of the $1 s$ oxygen core level. The calculated work functions are 5.79 $\mathrm{eV}$ and $5.68 \mathrm{eV}$ for the $\mathrm{O}-(2 \times 2) / \mathrm{Rh}(111)$ structures with $\mathrm{O}$ 
in an fcc and hep site at a 0.25 -ML coverage, respectively. That is, for the fcc-site adsorption the work-function change $\Delta \Phi$ (see Sec. III C) is largest. As already mentioned, a workfunction increase reflects the electron transfer from the substrate to the $\mathrm{O}$ adatom caused by the high electronegativity of O. Moreover, the calculated O $1 s$ core energies with respect to the Fermi level such that they are aligned in energy show that the $1 s$ core level for $\mathrm{O}$ at the fcc site is less bound by $0.36 \mathrm{eV}$. This initial-state effect reflects the larger electrostatic repulsion at the fcc site, where a somewhat more negatively charged $\mathrm{O}$ atom is adsorbed. $\mathrm{O}$ finds fcc hollows on $\mathrm{Rh}(111)$ favorable because of a stronger ionic bonding.

As the coverage increases, the binding energy per $\mathrm{O}$ atom decreases, but for all coverages $\mathrm{O}_{2}$ dissociation is exothermic. Such a behavior reflects the repulsive adsorbateadsorbate interactions with decreasing $\mathrm{O}-\mathrm{O}$ distance. Thus, the high-coverage $(1 \times 1)$ adlayer structure is stable by 1.40 $\mathrm{eV} /$ atom and may be able to form. However, as mentioned in the Introduction, under UHV conditions experiments indicate that the $(2 \times 1)$ phase is the terminal one. Therefore, the formation of such a dense structure by exposing the surface to $\mathrm{O}_{2}$ may be hindered by energy barriers for the oxygen dissociation.

A notable difference between $\mathrm{O}$ adsorption on $\mathrm{Rh}(111)$ and $\mathrm{Ru}(0001)$ is that for the latter the predicted stability of a high-coverage $(1 \times 1)$ phase by DFT calculations ${ }^{10}$ has been confirmed experimentally. ${ }^{11}$ The calculated adsorption energy for the stable $\mathrm{O}-(1 \times 1) / \mathrm{Ru}(0001)$ with $\mathrm{O}$ on the hcphollow sites is larger by $\sim 1.8 \mathrm{eV} /$ atom than that which $\mathrm{O}$ atoms have in $\mathrm{O}_{2} \cdot{ }^{10}$ Room-temperature exposure of $\mathrm{Rh}(111)$ and $\mathrm{Ru}(0001)$ to $\mathrm{O}_{2}$ leads to saturated atomic oxygen layers associated with the formation of $\mathrm{O}-(2 \times 1)$ phases. However, as mentioned above, exposure of the $\mathrm{O}-(2 \times 1) / \mathrm{Ru}(0001)$ to $\mathrm{NO}_{2}$ at a sample temperature of $600 \mathrm{~K}$ resulted in an O- $(1 \times 1)$ ordered adlayer. ${ }^{11}$ The same approach was tried in order to reach high $\mathrm{O}$ coverages on $\mathrm{Rh}(111)$, but in contrast to $\mathrm{Ru}(0001)$, before the half-order LEED spots faded away completely, additional spots indicated a $\sim(7 \times 7)$ lattice. ${ }^{20} \mathrm{~A}$ similar result was previously reported by Castner and Somorjai. ${ }^{17}$ After heating a $\mathrm{Rh}(111)$ sample in a 1-torr $\mathrm{O}_{2}$ pressure at $975 \mathrm{~K}$ for $10 \mathrm{~min}$, they observed an $(8 \times 8)$ pattern, which they assigned to an epitaxially grown $\mathrm{Rh}_{2} \mathrm{O}_{3}(0001)$ surface oxide on the $\mathrm{Rh}(111)$ substrate.

Moreover, a recent x-ray photoelectron diffraction (XPD) study of the $\mathrm{O} / \mathrm{Rh}(111)$ system, after exposure to $10^{5} \mathrm{LO}_{2}$ at a surface temperature of $470 \mathrm{~K}$, indicates that $\sim 0.05 \mathrm{ML}$ of subsurface oxygen can be stabilized on $\mathrm{Rh}(111)$, while the concentration of the adsorbed species rises to $0.83 \mathrm{ML}^{28}$ Similarly, a few percent of subsurface oxygen species was observed on $\mathrm{Ru}(0001)$ after dosing $1.5 \times 10^{6} \mathrm{~L}$ of $\mathrm{O}_{2}$ at a temperature of about $450 \mathrm{~K} .{ }^{12}$ However, the concentration of the adsorbed on-surface species was $1 \mathrm{ML}$. At about $600 \mathrm{~K}$ the oxygen uptake increased steeply due to further penetration of oxygen into the subsurface region and substantially higher total coverages were achievable.

We are presently investigating the stability of subsurface oxygen on $\mathrm{Rh}(111)$ as a function of on-surface coverage. Our calculations indicate, for example, that the occupation of the subsurface octahedral site in the $(2 \times 2)$ structure, just below the first $\mathrm{Rh}$ layer, is still exothermic for an on-surface coverage of $0.75 \mathrm{ML}$; however, appreciably less favorable than
TABLE V. Binding energies, $E_{b}$ [Eq. (1)], in eV/atom for the adlayers structures investigated, with $\mathrm{O}$ at fcc-hollow sites.

\begin{tabular}{cccc}
\hline \hline Structure & Coverage & Present work & Ref. 24 \\
\hline O- $(2 \times 2)$ & 0.25 & 5.22 & 5.03 \\
O- $(2 \times 1)$ & 0.5 & 4.93 & 4.85 \\
O- $(1 \times 1)$ & 1 & 4.38 & 4.24 \\
\hline \hline
\end{tabular}

the on-surface sites for a 1.0 ML coverage. Thus, we argue that the existence of a dense $\mathrm{O}$ overlayer with a $(1 \times 1)$ periodicity is possible because oxygen will be incorporated as a subsurface adsorbate only when the $(1 \times 1)$ adlayer is (nearly) completed. The results will be discussed in a future paper.

Our calculated binding energies $E_{b}(\Theta)=E_{\mathrm{ad}}(\Theta)+D / 2$ of $\mathrm{O}$ on $\mathrm{Rh}(111)$ are compared to those of Loffreda et $\mathrm{al}^{24}$ in Table V. The largest quantitative difference with our results is as much as $\sim 0.2 \mathrm{eV} /$ atom. The agreement seems reasonable if we consider the number of different approximations involved in the calculations of Ref. 24 compared to the present study, in particular, the use of the LDA exchange and correlation potential of Vosko-Wilko-Nusair for the wavefunction calculation and the nonlocal gradient corrections (Becke 88 for the exchange and Perdew 86 for the correlation energies) for the energies. The atoms were modeled by a frozen core, and relativistic effects were not included in the calculations. Moreover, no relaxation of the clean and oxygen-covered surfaces was considered, and the experimental lattice constant had been used to set up the slabs geometries with $\mathrm{O}$ placed at the bond length optimized for the lowest-coverage (unrelaxed) structure studied. Although in Ref. 24 most of the above different approximations were tested and no change in the general trend has been reported; the errors they introduce may add to some degree to account for the quantitative difference between those and the present results.

Temperature programmed desorption (TPD) is generally a powerful method for extracting activation energies for desorption for adsorbate systems. However, the data for oxygen on rhodium surfaces are often contradictory or inconsistent, which seems to be mainly due to the difficulty of cleaning the surface and keeping it clean. In particular, residues of carbon react with the oxygen at high temperature and remove intensity from the signal, while adsorption of either hydrogen or $\mathrm{CO}$ from the residual gas also carries away oxygen. ${ }^{2}$ Thus, the results of the analyses of thermaldesorption data for the activation energy for oxygen desorption from $\mathrm{O} / \mathrm{Rh}$ systems should be regarded with caution and be taken only as a qualitative guide to bond strengths.

TPD studies of $\mathrm{O} / \mathrm{Rh}(111)$ reported data for the activation energy for desorption at low coverages yield values 2.43 $\pm 0.10 \mathrm{eV} / \mathrm{O}_{2}$ to $3.69 \pm 0.20 \mathrm{eV} / \mathrm{O}_{2} .{ }^{4,27,51}$ While part of these discrepancies may be due to differences in heating rate, the differences seem a bit too large to be explained completely in this manner. The consistent disappearance of oxygen from the TPD spectra of Thiel et al., ${ }^{51}$ even though it was visible in the Auger spectra, suggests that there might have been a problem of $\mathrm{CO}$ adsorption from the residual gas or of carbon contamination. ${ }^{52}$ Nevertheless, the proposed experimental values for the heat of desorption seem to suggest 
somewhat smaller values for the $\mathrm{O}$ adsorption energy (1.215-1.845 eV/atom) than our calculated value of $2.24 \mathrm{eV} /$ atom at $\Theta=0.25 \mathrm{ML}$.

Density-functional theory within the generalized gradient approximation has demonstrated that the calculated bond energies of molecules, the cohesive energies of solids, atomic and molecular adsorption energies, and the energy barriers for molecular reactions are overestimated. ${ }^{50}$ In particular, the energy scale that is relevant when comparing the densityfunctional theory within the GGA of Perdew et al. ${ }^{29}$ derived atomic binding energies [see Eq. (1)] with experimental values is $0.25-1.0 \mathrm{eV} /$ atom. ${ }^{50}$ For adsorption energies [see Eq. (2)], we expect somewhat less severe deviations because $\mathrm{O}$ atomic adsorption $\left(E_{\mathrm{ad}}\right)$ and binding $\left(E_{b}\right)$ energies differ by half the dissociation energy of $\mathrm{O}_{2}$, which is also overestimated in DFT-GGA calculations. The calculated value, 2.24 eV/atom compares particularly poorly with the value extracted from Ref. 51, $1.215 \mathrm{eV} /$ atom but presumably the disagreement is due to the sample condition of that experimental study as explained.

Further inspection of Fig. 8, which compares the calculated adsorption energies of $\mathrm{O}$ adlayers on $\mathrm{Rh}(111)$ (top) with the corresponding calculated values for the $\mathrm{O} / \mathrm{Ru}(0001)$ surfaces (bottom) of Ref. 10, shows that oxygen on $\mathrm{Rh}(111)$ at the most stable fcc-hollow site binds less stronger than on $\mathrm{Ru}(0001)$ at the hcp-hollow site, at all coverages. This is a direct consequence of the dependence of the strength of the oxygen-metal bond with the relative position of the center of the clean metal surface $d$ band. In the case of Rh compared to $\mathrm{Ru}$, additional antibonding states are populated near the Fermi level, and hence the binding is weaker (see, e.g., Ref. $53)$.

\section{SUMMARY}

In summary, we have shown that adsorption of oxygen on $\mathrm{Rh}(111)$ gives rise to different stable structures, namely $\mathrm{O}-(2 \times 2)$, $\mathrm{O}-(2 \times 1)$, and $\mathrm{O}-(1 \times 1)$ at coverages $0.25,0.5$, and 1.0 ML, respectively. On the $\mathrm{Rh}(111)$ surface at all coverages, the fcc-hollow site is the most stable over the hcphollow site. This is due to the stronger ionic bonding of $\mathrm{O}$ at the fcc sites. The prediction about the existence of a dense $\mathrm{O}$ overlayer (close to $\Theta=1)$ with a $(1 \times 1)$ periodicity is based on the fact that oxygen will be incorporated as a subsurface adsorbate only when the $(1 \times 1)$ adlayer is (nearly) completed. ${ }^{54}$ The structural and electronic properties of the $\mathrm{O} / \mathrm{Rh}(111)$ system have been investigated as a function of oxygen on-surface coverage, $\Theta$. In particular, we have discussed the oxygen-induced increase of the average spacing between the outermost substrate layers, which shows a strong oxygen coverage dependence. This is illustrated in Fig. 4. On $\mathrm{Rh}(111)$ a high electronegative $\mathrm{O}$ adatom interacts with its nearest-neighbor metal atoms. Consequently, electron density is reduced between the two outermost substrate layers. Thus, Rh-Rh bonds get weakened giving rise to the oxygen-induced increase of the spacing between the outermost substrate layers. The calculated work-function change as a function of $\Theta$ reflects the high electronegativity of $\mathrm{O}$, which results in an induced inward dipole moment. This is illustrated in Figs. 5-7.

A number of similarities and differences in adsorption behavior between $\mathrm{Rh}(111)$ and neighboring $\mathrm{Ru}(0001)$ surfaces have been discussed. For instance, $\mathrm{O}$ on $\mathrm{Ru}(0001)$ at all coverages occupies hcp-hollow sites, ${ }^{10}$ the sites a next substrate layer would occupy, a difference over $\mathrm{O} / \mathrm{Rh}(111)$ that can be traced back to the same effect that stabilizes the fcc structure of bulk $\mathrm{Rh}$ and hcp of bulk Ru. Moreover, the oxygen-induced effect on the spacing between the outermost $\mathrm{Ru}(0001)$ substrate layers relative to the bulk interlayer spacing is less pronounced. These effects can be traced back to the higher cohesive energy of $\mathrm{Ru}$ over $\mathrm{Rh}$. Finally, $\mathrm{O}$ binds less strongly at the most stable fcc sites on $\mathrm{Rh}(111)$ than at the hcp sites on $\mathrm{Ru}(0001)$. This relates to the relative position of the surface $d$-band center of the clean metal surfaces.

In order for the theory to help define precisely the detailed nature of subsurface oxygen and surface oxides and unravel the role of the interaction between $\mathrm{O}$ with $\mathrm{Rh}$ and $\mathrm{Ru}$ surfaces in the apparent ease of formation of unreactive surface oxides on Rh surfaces, it is necessary to go beyond the adlayer systems; that is, to explicitly consider these other states of oxygen. An effort to do so is our intention in future work. ${ }^{54}$

Note added in proof. We have learned of the work of Gibson et al., ${ }^{55}$ who recently demonstrated that the $\mathrm{Rh}(111)$ surface is capable of supporting a full monolayer of atomic $\mathrm{O}$ when using atomic $\mathrm{O}$ for dosing. Oxygen is adsorbed as a well-ordered $(1 \times 1)-\mathrm{O}$ overlayer, as shown by $\mathrm{He}$ atom diffraction.

\section{ACKNOWLEDGMENTS}

We are grateful to A. Seitsonen, A. Fahmi, C. Stampfl, and $\mathrm{H}$. Over for useful suggestions and discussions. We thank S. Schwegmann for detailed information on his LEED data. We also thank M. Petersen, P. Blaha, M. Fuchs, A. Baraldi, P. Brault, and H. Weinberg for helpful discussions. We thank P. Marcus for a careful reading of the manuscript.

\section{APPENDIX}

To test the accuracy of our calculated adsorption energies on a number of approximations, namely, the cutoff energy of the plane-wave basis set $E_{\text {cut }}$, the slab thickness, and the number of $\mathbf{k}$ points to sample the Brillouin zone, selected calculations were repeated with more accuracy. We find that none of these affect the conclusions made in this work. In the following, the results of these tests will be present as differences $(\Delta E)$ from the results previously discussed in the text. In particular, because $E_{\text {ad }}=E_{b}-D / 2$ and the calculation of $D$ was performed using a higher $E_{\text {cut }}, \Delta E_{\text {ad }}=\Delta E_{b}$. One should, however, note that the calculation of $E_{b}$ according to Eq. (1) involves the calculation of $E_{\mathrm{O}, \mathrm{Rh}}, E_{\mathrm{Rh}}$, and $E_{\mathrm{O}}$, which depend on the choice of $E_{\text {cut }}$.

(i) The cutoff energy of the plane-wave basis set, $E_{\text {cut }}$. The present self-consistent structure optimizations and binding-energy calculations were conducted for a 16-Ry cutoff. In Fig. 10 we show the effect on the calculated binding energy of the $(1 \times 1)$ structure with $\mathrm{O}$ adatoms on fcc sites as a function of $E_{\text {cut }}$. We neglect changes due to further atomic relaxations, i.e., the atomic positions have been fixed in their stable positions of the $E_{\text {cut }}=16-\mathrm{Ry}$ structure optimization. Inclusion of such relaxations causes changes in the binding 


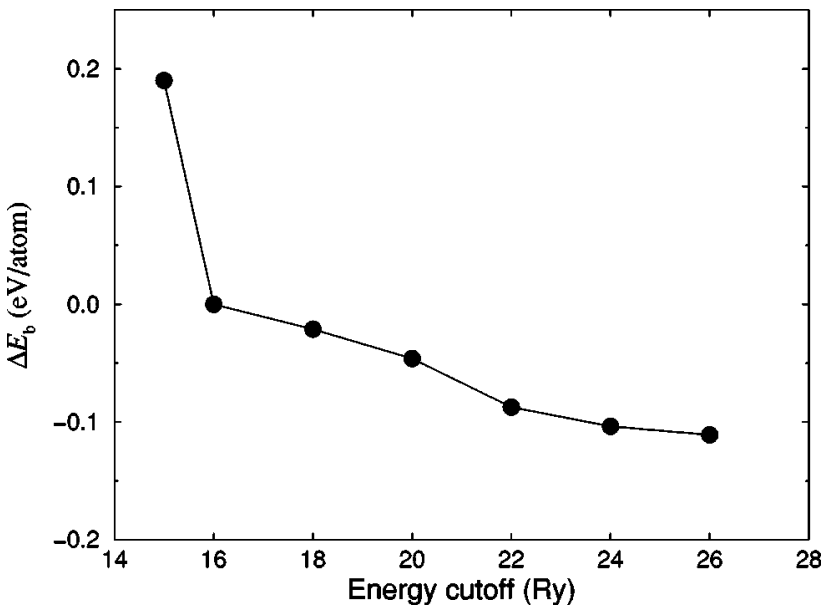

FIG. 10. Calculated binding energy differences, $\Delta E_{b}$ in $\mathrm{eV} /$ atom, for $\mathrm{O}$ in the fcc-hollow site and in the $(1 \times 1)$ adlayer structure as a function of the plane-wave expansion energy cutoff, $E_{\text {cut }}$. Calculations have been performed at the stable geometry of the 16-Ry structure optimization and the other FP-LAPW parameters as in Sec. II. Differences are referred to the 16-Ry self-consistent calculation.

energies by less than $0.01 \mathrm{eV} / \mathrm{atom}$. This shows that the chosen $E_{\text {cut }}=16$ Ry gives a good estimate of the stable geometries and that the error of the binding energy of $\mathrm{O}$ on $\mathrm{Rh}(111)$ at a coverage $\Theta=1.0 \mathrm{ML}$ is less than $\approx 0.1 \mathrm{eV} /$ atom. Finally, to determine if this overestimation of the calculated binding energy $\Delta E_{b}$ induced by the use of a 16-Ry $E_{\text {cut }}$ is independent of coverage, we have investigated for $\mathrm{O}$ in fcc sites and different coverages the effect on the calculated binding energies due to a cutoff decrease from 16 to $15 \mathrm{Ry}$ and an increase from 16 to $24 \mathrm{Ry}$ (calculations performed for the 16-Ry stable geometries).

The overestimation $\Delta E_{b}$ of a 15-Ry against a $16-\mathrm{Ry}$ calculation is $\approx 0.2 \mathrm{eV} /$ atom independent of the coverage, as shown in Table VI. Similarly, the comparison of the calculated binding energies of a 24-Ry against a 16-Ry calculation for the higher-coverage structures (and the 16-Ry optimized
TABLE VI. Comparison of the calculated adsorption energies, $E_{\text {ad }}$ in eV/atom for the adlayers structures investigated as a function of $E_{\text {cut }}$, given as differences from the values obtained with a 16-Ry self-consistent calculation. Calculations for $15 \mathrm{Ry}$ and $24 \mathrm{Ry}$ were performed at the stable geometries of the 16-Ry structure optimizations.

\begin{tabular}{ccccc}
\hline \hline Structure & Coverage & $16 \mathrm{Ry}$ & $15 \mathrm{Ry}$ & $24 \mathrm{Ry}$ \\
\hline $\mathrm{O}-(2 \times 2)$ & 0.25 & 0.0 & +0.177 & \\
$\mathrm{O}-(2 \times 1)$ & 0.5 & 0.0 & +0.185 & -0.060 \\
$\mathrm{O}-(1 \times 1)$ & 1 & 0.0 & +0.190 & -0.104 \\
\hline \hline
\end{tabular}

geometries) gives values essentially independent of the coverage $(\approx 0.1 \mathrm{eV} /$ atom $)$. These results demonstrate that the $\Theta$ dependence of the error induced by the use of a 16-Ry $E_{\text {cut }}$ is weak. The reason for the improvement of the calculated overbinding can be attributed largely to a systematic improvement in the description of both the free oxygen atom and the oxygen-covered surface. However, the error itself is the result of a less accurate total-energy prediction for the free $\mathrm{O}$ atom for lower cutoff energies. Thus the calculated binding energies decrease as $E_{\text {cut }}$ increases.

(ii) We approximate the surfaces by slabs of finite thickness with $\mathrm{O}$ adatoms on both sides of the slabs. Extending the $\mathrm{Rh}(111)$ from 7 to 9 and 11 close-packed layers weakens the bond by 4 and $9 \mathrm{meV} / \mathrm{atom}$, respectively.

(iii) The Brillouin-zone integration has been performed using a $(12 \times 12 \times 1)$ Monkhorst-Pack grid of special points for the $(1 \times 1)$ surface unit cell, which corresponds to $180 \mathbf{k}$ points in the whole zone. For the $(2 \times 1)$ and $(2 \times 2)$ surface unit cells, grids have been chosen so as to obtain the same sampling of the reciprocal space. Tests with 360 and $540 \mathbf{k}$ points show that the convergence error for the binding energies of the $(1 \times 1)$ structure was lower than $0.01 \mathrm{eV} / \mathrm{atom}$.

Thus the above discussed approximations are found to be negligible on the scale that is relevant when comparing the density-functional-theory-derived adsorption energies with experimental values.
${ }^{1}$ U. Starke, M. A. Van Hove, and G. A. Somorjai, Prog. Surf. Sci. 46, 305 (1994).

${ }^{2}$ See G. Commelli, V. R. Dhanak, M. Kiskinova, K. C. Prince, and R. Rosei, Surf. Sci. Rep. 32, 165 (1998), and references therein.

${ }^{3}$ F. Besenbacher and J. Nørskov, Prog. Surf. Sci. 44, 5 (1993).

${ }^{4}$ K. A. Peterlinz and S. J. Sibener, J. Phys. Chem. 9, 2817 (1995).

${ }^{5}$ J. Segner, C. T. Campbell, G. Doyen, and G. Ertl, Surf. Sci. 138, 505 (1998).

${ }^{6}$ M. Rebholz, R. Prins, and N. Kruse, Surf. Sci. 269/270, 293 (1992).

${ }^{7}$ V. A. Bondzie, P. Kleban, and D. J. Dwyer, Surf. Sci. 347, 319 (1996).

${ }^{8}$ A. von Oertzen, A. Mikhailov, V. H. Rotermund, and G. Ertl, Surf. Sci. 350, 259 (1996).

${ }^{9}$ N. M. H. Janssen, A. Schaak, B. E. Nieuwenhuys, and R. Imbihl, Surf. Sci. 364, L555 (1996).

${ }^{10}$ C. Stampfl and M. Scheffler, Phys. Rev. B 54, 2868 (1996).

${ }^{11}$ C. Stampfl, S. Schwegmann, H. Over, M. Scheffler, and G. Ertl,
Phys. Rev. Lett. 77, 3371 (1996).

${ }^{12}$ A. Böttcher, H. Niehus, S. Schwegmann, H. Over, and G. Ertl, J. Phys. Chem. B 101, 11185 (1997).

${ }^{13}$ C. H. F. Peden, in Surface Science of Catalysis: In Situ Probes and Reaction Kinetics, edited by D. J. Dwyer and F. M. Hoffman (American Chemical Society, Washington, D.C., 1992).

${ }^{14}$ C. H. F. Peden, D. W. Goodman, D. S. Blair, P. J. Berlowitz, G.

B. Fischer, and S. H. Oh, J. Phys. Chem. 92, 1563 (1988).

${ }^{15}$ S. H. Oh and J. E. Carpenter, J. Catal. 80, 472 (1983).

${ }^{16}$ J. T. Grant and T. W. Haas, Surf. Sci. 21, 70 (1970).

${ }^{17}$ D. G. Castner, B. A. Sexton, and G. A. Somorjai, Surf. Sci. 71, 519 (1978); D. G. Castner and G. A. Somorjai, ibid. 83, 60 (1979); Appl. Surf. Sci. 6, 29 (1980).

${ }^{18}$ K. C. Wong, K. C. Hui, M. Y. Zhou, and K. A. R. Mitchell, Surf. Sci. 165, L21 (1986).

${ }^{19}$ K. C. Wong, W. Liu, and K. A. R. Mitchell, Surf. Sci. 360, 137 (1996). 
${ }^{20}$ S. Schwegmann, H. Over, V. De Renzi, and G. Ertl, Surf. Sci. 375, 91 (1997).

${ }^{21}$ S. Schwegmann and H. Over, Surf. Sci. 393, 179 (1997); H. Xu and K. Y. S. Ng, ibid. 393, 181 (1997).

${ }^{22}$ H. Xu and K. Y. S. Ng, Surf. Sci. 375, 161 (1997).

${ }^{23}$ M. Chen, S. P. Bates, R. A. van Santen, and C. M. Friend, J. Phys. Chem. B 101, 10051 (1997).

${ }^{24}$ D. Loffreda, D. Simon, and P. Sautet, J. Chem. Phys. 108, 6447 (1998).

${ }^{25}$ C. T. Reimann, M. El-Maazawi, K. Walzl, B. J. Garrison, N. Winograd, and D. M. Deaven, J. Chem. Phys. 90, 2027 (1989).

${ }^{26}$ T. Greber, J. Wider, E. Wetli, and J. Osterwalder, Phys. Rev. Lett. 81, 1654 (1998).

${ }^{27}$ T. W. Root, L. D. Schmidt, and G. B. Fisher, Surf. Sci. 134, 30 (1983).

${ }^{28}$ J. Wider, T. Greber, E. Wetli, T. J. Kreutz, P. Schwaller, and J. Osterwalder, Surf. Sci. 417, 301 (1998).

${ }^{29}$ J. P. Perdew, J. A. Chevary, S. H. Vosko, K. A. Jackson, M. R. Pederson, D. J. Singh, and C. Fiolhais, Phys. Rev. B 46, 6671 (1992).

${ }^{30}$ P. Blaha, K. Schwarz, and J. Luitz, wIEN97 (Vienna University of Technology, Vienna, 1997). [Improved and updated Unix version of the original copyrighted WIEN code, which was published by P. Blaha, K. Schwarz, P. Sorantin, and S. B. Trickey, Comput. Phys. Commun. 59, 399 (1990)].

${ }^{31}$ M. Petersen, F. Wagner, L. Hufnagel, M. Scheffler, P. Blaha, and K. Schwarz, Comput. Phys. Commun. (to be published).

${ }^{32}$ B. Kohler, S. Wilke, M. Scheffler, R. Kouba, and C. AmbroschDraxl, Comput. Phys. Commun. 94, 31 (1996).

${ }^{33}$ P. Villars and L. D. Calvert, Pearson's Handbook of Crystallographic Data for Intermetallic Phases (ASM, Metals Park, $\mathrm{OH}$, 1985).

${ }^{34}$ C. Kittel, Introduction to Solid State Physics, 6th ed. (Wiley, New York, 1986), p. 55.

${ }^{35}$ A. Eichler, J. Hafner, and G. Kresse, J. Phys.: Condens. Matter 8, 7659 (1996).

${ }^{36}$ J. Xie and M. Scheffler, Phys. Rev. B 57, 4768 (1998).

${ }^{37}$ A. Barbieri, M. A. Van Hove, and G. A. Somorjai, Surf. Sci. 306, 261 (1994).
${ }^{38}$ M. Methfessel, D. Hennig, and M. Scheffler, Phys. Rev. B 46, 4816 (1992).

${ }^{39}$ A. Eichler, J. Hafner, J. Furthmüller, and G. Kresse, Surf. Sci. 346, 300 (1996).

${ }^{40}$ M. Lindroos, H. Pfnür, G. Held, and D. Menzel, Surf. Sci. 222, 451 (1989); H. Pfnür, G. Held, M. Lindroos, and D. Menzel, ibid. 220, 43 (1989).

${ }^{41}$ H. L. Skriver, Phys. Rev. B 31, 1909 (1985).

${ }^{42}$ CRC Handbook of Chemistry and Physics, 76th ed., edited by D. R. Lide (CRC Press, Boca Raton, FL, 1995), pp. 12-123.

${ }^{43}$ P. Brault, H. Range, J. P. Toennies, and Ch. Wöll, Z. Phys. Chem. (Munich) 198, 1 (1997).

${ }^{44}$ L. A. Curtiss, K. Raghavachari, G. W. Trucks, and J. A. Pople, J. Chem. Phys. 94, 7221 (1991).

${ }^{45}$ K. P. Huber and G. Herzberg, Molecular Spectra and Molecular Structure IV. Constants of Diatomic Molecules (Van Nostrand, Princeton, 1979); Numerical Data and Functional Relationships in Science and Technology, edited by K. H. Hellwege and A. M. Hellwege, Landolt-Börnstein, New Series, Group II, Vol. 7 (Springer, Berlin, 1979).

${ }^{46}$ A. D. Becke, J. Chem. Phys. 96, 2155 (1992); 97, 9173 (1992).

${ }^{47}$ E. I. Proynov, A. Vela, and D. R. Salahub, Chem. Phys. Lett. 230, 419 (1994).

${ }^{48}$ J. P. Perdew, K. Burke, and M. Ernzerhof, Phys. Rev. Lett. 77, 3865 (1996).

${ }^{49}$ Y. Zhang and W. Yang, Phys. Rev. Lett. 80, 890 (1998).

${ }^{50}$ B. Hammer, L. B. Hansen, and J. K. Nørskov, Phys. Rev. B 59, 7413 (1999).

${ }^{51}$ P. A. Thiel, J. T. Yates, Jr., and W. H. Weinberg, Surf. Sci. 82, 22 (1979).

${ }^{52}$ G. B. Fisher and S. J. Schmieg, J. Vac. Sci. Technol. A 1, 1064 (1983).

${ }^{53}$ B. Hammer and J. K. Nørskov, in Chemisorption and Reactivity on Supported Clusters and Thin Films, edited by R. M. Lambert and G. Pachioni (Kluwer Academic, The Netherlands, 1997), pp. 285-351.

${ }^{54}$ M. V. Ganduglia-Pirovano and M. Scheffler (unpublished).

${ }^{55}$ K. D. Gibson, M. Viste, E. C. Sanchez, and S. J. Sibener, J. Chem. Phys. 110, 2757 (1999). 